\title{
A CIRCULAR PROPERTY OF THE OCCURRENCE OF SEQUENCE PATTERNS IN THE FAIR COIN-TOSSING PROCESS
}

\author{
ROBERT W. CHEN, ${ }^{*}$ University of Miami
}

Let $\Omega=\{0,1\}$ and for each integer $n \geqq 1$ let $\Omega_{n}=\Omega \times \Omega \times \cdots \times \Omega$ (n-tuple) and $\Omega_{n}^{k}=\left\{\left(a_{1}, a_{2}, \cdots, a_{n}\right) \mid\left(a_{1}, a_{2}, \cdots, a_{n}\right) \in \Omega_{n}\right.$ and $\left.\sum_{i=1}^{n} a_{i}=k\right\}$ for all $k=0,1, \cdots, n$. Let $\left\{X_{m}\right\}_{m \geqq 1}$ be a sequence of i.i.d. random variables such that $P\left(X_{1}=0\right)=P\left(X_{1}=1\right)=\frac{1}{2}$. Any element $A$ in $\Omega_{n}$ is called a sequence pattern and for each sequence pattern $A$, let $T_{A}$ be the first occurrence time of $A$ (with respect to the process $\left\{X_{m}\right\}_{m \geqq 1}$ ) which is defined by $T_{A}\left(X_{1}, X_{2}, \cdots\right)=\inf \left\{m \mid\left(X_{m-n+1}, \cdots, X_{m}\right)=A\right\}$. For any two distinct sequence patterns $A$ and $B, A$ occurs (stochastically) after $B$ (denoted by $B \ll A$ ) if $P\left(T_{A}>T_{B}\right)>\frac{1}{2}$. In this paper, we prove that if $n \geqq 4, k=1,2,3(n-3, n-2, n-1)$, and $n \neq 2 k$, then there is an arrangement $\left\{B_{1}, B_{2}, \cdots, B_{\left(\begin{array}{c}n \\ k\end{array}\right)}\right\}$ of $\Omega_{n}^{k}$ such that $B_{1} \ll B_{2} \ll \cdots \ll B_{\left(\begin{array}{c}n \\ k\end{array}\right)} \ll B_{1}$. Albeit we are not able to prove the statement for any $k \neq n / 2$, from our proof for special cases we strongly believe that the statement is true for any $n \geqq 4$ and $k \neq n / 2$. This new result reveals a circular property of the first occurrence among the sequence patterns in $\Omega_{n}^{k}$ and also provides us with an understanding of the regularity of the fair coin-tossing process. We start with the following notation and lemmas.

For each sequence pattern $A=\left(a_{1}, a_{2}, \cdots, a_{n}\right)$ and $B=\left(b_{1}, b_{2}, \cdots, b_{n}\right)$ (not necessarily distinct) in $\Omega_{n}$, we define $A * B=\sum_{j=1}^{n} 2^{j} \varepsilon_{j}$, where for each $j=1,2, \cdots, n, \varepsilon_{j}=1$ or 0 (according to whether $\left(b_{1}, b_{2}, \cdots, b_{j}\right)=\left(a_{n-j+1}, \cdots, a_{n}\right)$ or not.

Lemma 1. If $A$ and $B$ are two distinct sequence patterns in $\Omega_{n}$, then $P\left(T_{A}<T_{B}\right) / P\left(T_{B}<\right.$ $\left.T_{A}\right)=(B * B-B * A) /(A * A-A * B)$.

Lemma 2. If $1 \leqq k \leqq n-1, k \neq n / 2, n \geqq 4$, and $A=\left(a_{1}, a_{2}, \cdots, a_{n}\right)$ is in $\Omega_{n}^{k}$, then $B \ll A$, where $B=\left(a_{n}, a_{1}, a_{2}, \cdots, a_{n-1}\right)$.

Theorem 1. If $n \geqq 4$, then there is an arrangement $\left\{B_{1}, B_{2}, \cdots, B_{n}\right\}$ of $\Omega_{n}^{1}\left(\right.$ or $\Omega_{n}^{n-1}$ ) such that $B_{1} \ll B_{2} \ll \cdots \ll B_{n} \ll B_{1}$.

For each $A=\left(a_{1}, a_{2}, \cdots, a_{n}\right)$ in $\Omega_{n}$, let $A^{1}=A$ and $A^{j+1}=\left(a_{n-j+1}, \cdots, a_{n}, a_{1}, \cdots, a_{n-j}\right)$ for all $j=1,2, \cdots, n-1$. For each $i=0,1, \cdots, n-2$, let $A_{i}=(1,0, \stackrel{\cdot}{\cdot}, 0,1,0, \cdots, 0)$.

Lemma 3. If $n=2 m+1$ and $i \leqq n-3$, then $A_{i+1}^{1} \ll A_{i}^{n}$ if $m \geqq 3$ or $m=2$ but $i \neq 1$.

Lemma 4. If $n=2 m+1 \geqq 5$ and $A=(1,1,0, \cdots, 0)$ is in $\Omega_{n}^{2}$, then $A \ll A_{i}^{n}$ for all $i=0,1,2, \cdots, n-2$.

Lemma 5. If $n=2 m+1 \geqq 7$, then there is an arrangement $\left\{B_{1}, B_{2}, \cdots, B_{\left(\begin{array}{c}n \\ 2\end{array}\right)}\right\}$ of $\Omega_{n}^{2}$ (or $\Omega_{n}^{n-2}$ ) such that $B_{1} \ll B_{2} \ll \cdots \ll B_{\left(\frac{n}{2}\right)} \ll B_{1}$.

Proof. Let $A_{i}$ and $A_{i}^{j}$ be as defined just above Lemma 3 for all $j=1,2, \cdots, n$ and $i=0,1, \cdots, n-2$. By Lemma $2, A_{i}^{j+1} \ll A_{i}^{j}$ for all $j=1,2, \cdots, n-1$ and all $i=$ $0,1, \cdots, m-1$. By Lemma $3, A_{i+1}^{1} \ll A_{i}^{n}$ for all $i=0,1, \cdots, m-1$ (since $m \geqq 3$ ). By Lemma $4, A_{0}^{1} \ll A_{m-1}^{n}$. Therefore, $A_{0}^{1} \ll A_{m-1}^{n} \ll \cdots \ll A_{m-1}^{1} \ll A_{m-2}^{n} \ll \cdots \ll A_{1}^{1} \ll A_{0}^{n} \ll$ $\cdots \ll A_{0}^{2} \ll A_{0}^{1}$. Since $n=2 m+1 \leqq 7$ and $\left\{A_{i}^{j} \mid 1 \leqq j \leqq n\right.$ and $\left.0 \leqq i \leqq m-1\right\}=\Omega_{n}^{2}$, the proof of Lemma 5 is now complete.

Received 17 January 1989; revision received 12 July 1989.

* Postal address: Department of Mathematics and Computer Science, University of Miami, P.O. Box 249085, Coral Gables, FL 33124, USA. 
Lemma 6. If $n=2 m \geqq 6$, then $A_{i+1}^{1} \ll A_{i}^{n}$ for all $i=0,1, \cdots, m-1$.

Lemma 7. If $n=2 m \geqq 6$, then there is an arrangement $\left\{B_{1}, B_{2}, \cdots B_{\left(\begin{array}{c}n \\ 2\end{array}\right)}\right\}$ of $\Omega_{n}^{2}\left(\right.$ or $\left.\Omega_{n}^{n-2}\right)$ such that $B_{1} \ll B_{2} \ll \cdots \ll B_{\left(\begin{array}{c}n \\ 2\end{array}\right)} \ll B_{1}$.

Proof. By Lemma $2, A_{i}^{j+1} \ll A_{i}^{j}$ for all $j=1,2, \cdots, n-1$ and all $i=0,1, \cdots, m-1$. By Lemma $6, A_{i+1}^{1} \ll A_{i}^{n}$ for all $i=0,1, \cdots, m-2$. Now by a direct computation, $A_{0}^{1} * A_{0}^{1}=$ $2^{2 m}, A_{0}^{1} * A_{m-1}^{m}=2^{m-1}, \quad A_{m-1}^{m} * A_{m-1}^{m}=2^{2 m}+2^{m}, \quad$ and $A_{m-1}^{m} * A_{0}^{1}=2$. Hence $A_{0}^{1} \ll A_{m-1}^{m}$. Therefore, $A_{0}^{1} \ll A_{m-1}^{m} \ll \cdots \ll A_{m-1}^{1} \ll A_{m-2}^{n} \ll \cdots \ll A_{m-2}^{1} \ll \cdots \ll A_{1}^{n} \ll \cdots \ll A_{1}^{1} \ll A_{0}^{n} \ll$ $\cdots \ll A_{0}^{2} \ll A_{0}^{1}$. Since $n=2 m \geqq 6$ and $\left\{A_{i}^{j} \mid 0 \leqq I \leqq m-2\right.$ and $\left.1 \leqq j \leqq n\right\} \cup\left\{A_{m-1}^{1}, \cdots, A_{m-1}^{m}\right\}=$ $\Omega_{n}^{2}$, the proof of Lemma 7 is now complete.

Theorem 2. If $n \geqq 5$, then there is an arrangement $\left\{B_{1}, B_{2}, \cdots, B_{\left(\begin{array}{c}n \\ 2\end{array}\right)}\right\}$ of $\Omega_{n}^{2}\left(\right.$ or $\left.\Omega_{n}^{n-2}\right)$ such that $B_{1} \ll B_{2} \ll \cdots \ll B_{\left(\begin{array}{c}n \\ 2\end{array}\right)} \ll B_{1}$.

Proof. If $n=5$, Theorem 2 can be proved by a direct computation and Lemma 1 . If $n \geqq 6$, Theorem 2 is proved by Lemmas 5 and 7.

For each $i=0,1, \cdots, n-3$, and $j=0,1, \cdots, n-3-i$, let $A_{i j}=(1,0, \overbrace{\bullet}^{i}, 0,1,0, \overbrace{\bullet}^{i}$, $0,1,0, \cdots, 0)$ be a sequence pattern in $\Omega_{n}^{3}$.

The following lemmas are essential to Theorem 3 below. However, the proofs of these lemmas are omitted.

Lemma 8. In $n \geqq 8$, then $A_{i+1,0}^{1} \ll A_{0, n-i-3}^{i+1}$ for all $i=0,1, \cdots, n-4$.

Lemma 9. If $n \geqq 8$, then $A_{11}^{1} \ll A_{01}^{n-3}$.

Lemma 10. If $n=3 m+3$ and $m \geqq 2$, then $A_{m, m}^{m} \ll A_{m, m-1}^{m+1}$.

Lemma 11. If $n=3 m+3$ and $m \geqq 1$, then $A_{m, m-1}^{m+2} \ll A_{m, m}^{1}$.

Lemma 12. If $n=3 m+3$ and $m \geqq 2$, then $A_{00}^{1} \ll A_{m+1, m}^{m}$.

Lemma 13. If $1 \leqq i \leqq n-2 i-7$, then $A_{i+1, i+1}^{1} \ll A_{n-2 i-4, i+1}^{i+1}$.

Lemma 14. If $1 \leqq i \leqq j$ and $i+1 \leqq n-i-j-3$, then $A_{00}^{1} \ll A_{j, n-i-j-3}^{i+1}$.

Lemma 15. If $1 \leqq i \leqq j$ and $i+2 \leqq n-i-j-3$, then $A_{i, j+1}^{1} \ll A_{j, n-i-j-3}^{i+1}$.

Theorem 3. If $n \geqq 7$, then there is an arrangement $\left\{B_{1}, B_{2}, \cdots, B_{\left(\frac{n}{3}\right)}\right\}$ of $\Omega_{n}^{3}\left(\right.$ or $\Omega_{n}^{n-3}$ ) such that $B_{1} \ll B_{2} \ll \cdots \ll B_{\left(\begin{array}{c}n \\ 3\end{array}\right)} \ll B_{1}$.

Proof. Since the case that $\left\{B_{1}, B_{2}, \cdots, B_{\left(\begin{array}{l}n \\ 3\end{array}\right)}\right\}=\Omega_{n}^{n-3}$ can be proved by interchanging 0 and 1 , we prove only the case that $\left\{B_{1}, B_{2}, \cdots, B_{\left(\frac{n}{3}\right)}\right\}=\Omega_{n}^{3}$. If $n=7$, then by a direct computation and Lemma 2 , the sequence patterns in $\Omega_{7}^{3}$ can be arranged as follows:

$$
\begin{aligned}
(1110000) & \ll(0101001) \ll(1010010) \ll(0100101) \ll(1001010) \ll(0010101) \\
& \ll(0101010) \ll(1010100) \ll(0001011) \ll(0010110) \ll(0101100) \ll(1011000) \\
& \ll(0110001) \ll(1100010) \ll(1000101) \ll(1001001) \ll(0010011) \ll(0100110) \\
& \ll(1001100) \ll(0011001) \ll(0110010) \ll(1100100) \ll(0001101) \ll(0011010) \\
& \ll(0110100) \ll(1101000) \ll(1010001) \ll(0100011) \ll(1000110) \ll(1100001) \\
& \ll(1000011) \ll(0000111) \ll(0001110) \ll(0011100) \ll(0111000) \ll(1110000)
\end{aligned}
$$

Now we assume that $k=3$ and $n \geqq 8$. When $n \neq 3 m+3$ for some positive integer $m$, Theorem 3 is proved by combining Lemmas $2,8,9,13,14$, and 15 . When $n=3 m+3$ for some positive integer $m$, Theorem 3 is proved by combining Lemmas $2,8,13,14,15$, and at certain steps, Lemmas 10, 11, 12 will be used to avoid the cyclic behavior. Since the detailed proof is very lengthy, we omit it. 
From our constructive proofs of Theorems 2 and 3, we strongly believe that Theorem 3 holds in general, i.e., if $n \geqq 4, k=1,2, \cdots, n-1$, and $k \neq n / 2$, then there is an arrangement $\left\{B_{1}, B_{2}, \cdots, B_{\left(\begin{array}{c}n \\ k\end{array}\right)}\right\}$ of $\Omega_{n}^{k}$ such that $B_{1} \ll B_{2} \ll \cdots \ll B_{\left(\begin{array}{c}n \\ k\end{array}\right)} \ll B_{1}$.

Chen and Lin (1984) proved that if $n \geqq 4$ and $n=2 k$, then $P\left(T_{A}<T_{A_{i}}\right)=\frac{1}{2}$ for $i=1,2$ and $P\left(T_{A}<T_{D}\right)<\frac{1}{2}$ for all $D \in \Omega_{2 k}^{k}-\left\{A, A_{1}, A_{2}\right\}$; here $A=(0,1,0,1, \cdots, 0,1), \quad A_{1}=$ $(1,0,1,0, \cdots, 1,0)$, and $A_{2}=(0,1,0,1, \cdots, 0,1,1,0)$. Hence there does not exist an arrangement $\left\{B_{1}, B_{2}, \cdots, B_{\left(\begin{array}{c}2 k \\ k\end{array}\right)}\right\}$ of $\Omega_{2 k}^{k}$ such that $B_{1} \ll B_{2} \ll \cdots \ll B_{\left(\begin{array}{c}2 k \\ k\end{array}\right)} \ll B_{1}$.

Under some mild conditions, the conjecture and the results in this paper can presumably be extended to the situation in which $\Omega=\{1,2, \cdots, r\}$ and $P\left(X_{1}=i\right)=1 / r$ for all $i=$ $1,2, \cdots, r$; here $r$ is a positive integer $\geqq 3$. Chen and Zame (1979) also briefly discussed this case.

\section{Acknowledgement}

We should like to thank Dr Frank K. Hwang for some useful conversation about the circular ordering among elements in a set. We also sincerely thank the referee for his invaluable comments.

\section{References}

Chen, R. ANd Lin, H. E. (1984) On the fair coin tossing process. J. Multivariate Anal. 15, 222-227.

Chen, R. AND ZAME, A. (1979) On fair coin-tossing games. J. Multivariate Anal. 9, 150-157. 